\title{
Neonatal Chylous Ascites Successfully Treated With MCT-Based Formula and Octreotide: A Case Report
}

\author{
Sanguansak Rerksuppaphol ${ }^{\mathrm{a}, \mathrm{c}}$, Lakkana Rerksuppaphol ${ }^{\mathrm{b}}$
}

\begin{abstract}
Neonatal chylous ascites is an anomalous development of intraabdominal lymphatic duct usually presents as a remedial challenge with symptomatic leaky lymphatics induced either as idiopathic or congenital or traumatic etiologies. In present case, female neonate developed abdominal distention on day 11 of life, just after introduction of enteral feeding. Chylous was diagnosed by paracenthesis and fluid examination. She was treated with TPN and followed by MCT based diet with octreotide. Baby was well tolerated with enteral feed and discharged on pregestimil on day 40 of life. On follow-up, baby had mild parenchymal liver disease with minimal ascites at right hepatic region at 4 months of age, which was completely resolved on 11 months. Discussed case suggests that octreotide with MCT oil based diet after TPN aids in repair of anomalous lymphatic vessel in presented rare instance of chylous ascites.
\end{abstract}

Keywords: Chylous ascites; Paracenthesis; MCT oil; Octreotide; Total parenteral nutrition; Thai neonat

\section{Introduction}

Neonatal chylous ascites is a rare condition that is scantly

Manuscript accepted for publication August 23, 2011

\footnotetext{
a Department of Pediatrics, Faculty of Medicine, Srinakharinwirot University, Thailand

${ }^{\mathrm{b}}$ Department of Preventive Medicine, Faculty of Medicine, Srinakharinwirot University, Thailand

${ }^{\mathrm{c} C}$ Corresponding author: Sanguansak Rerksuppaphol: Department of Pediatrics, Faculty of Medicine, Srinakhariwirot University, Rangsit-Nakorn Nayok Rd., NakornNayok, 26120, Thailand.

Email: sanguansak_r@hotmail.com
}

doi:10.4021/ijcp102e reported in worldwide literature. Chylous ascites is characterized by the accumulation of a milky or creamy fluid rich in triglycerides in the abdominal cavity. Data on pathogenesis and treatment options of neonatal chylous ascites are limited, however, a review from older children showed that trauma, obstruction and lymphatic abnormalities are the predisposing causes [1]. Available treatment options which usually reported from (older) children are TPN, diet modification, somatostatin or fibrin glue. As a surgical option, paracentesis or ligations are also used in a refractory case [2]. This case report describes about a neonatal chylous ascites and management of the same in the early days of nascence of Thai neonate.

\section{Case Report}

A female Thai infant was delivered at 36 weeks gestational age via caesarean section, because her mother had developed preeclampsia. At birth she weighed approximately 3,610 g, and her Apgar Scores were 8 and 10. Eleven hours after she was born, she developed tachypnea and was subsequently admitted to the neonatal intensive care unit (NICU). A chest $\mathrm{x}$-ray that was taken upon admission to the NICU to rule out respiratory distress syndrome and pneumonia revealed ground glass opacities and hypoaeration of the patient's lungs. The patient was subsequently intubated and placed on a ventilator setting with Patient Triggered Ventilation (PTV) mode. On ventilation, pulmonary functions were RR-60, Positive Inspiratory Pressure (PIP)-18, Positive End Expiratory Pressure (PEEP)4, Fraction of Inspired Oxygen (FiO2)-1 and Ti-0.35 second. On day 2 of life, echocardiogram exhibited patent ductus arteriosus (PDA) of $1.3 \mathrm{~mm}$ with mild pulmonary hypertension. She was restricted on fluid with intermittent diuretics. Empirical treatment with ampicillin and gentamycin was given for 5 days with provisional diagnosis of neonatal sepsis. While confirming the same on $\mathrm{H} / \mathrm{C}$, no growth was cultivated in 7 days. Ventilator was weaned off at day 4 and extubation was done at day 7. Umbilical venous catheter (UVC) line was taken off at day 21 of life and umbilical arterial catheter (UAC) line was taken off 


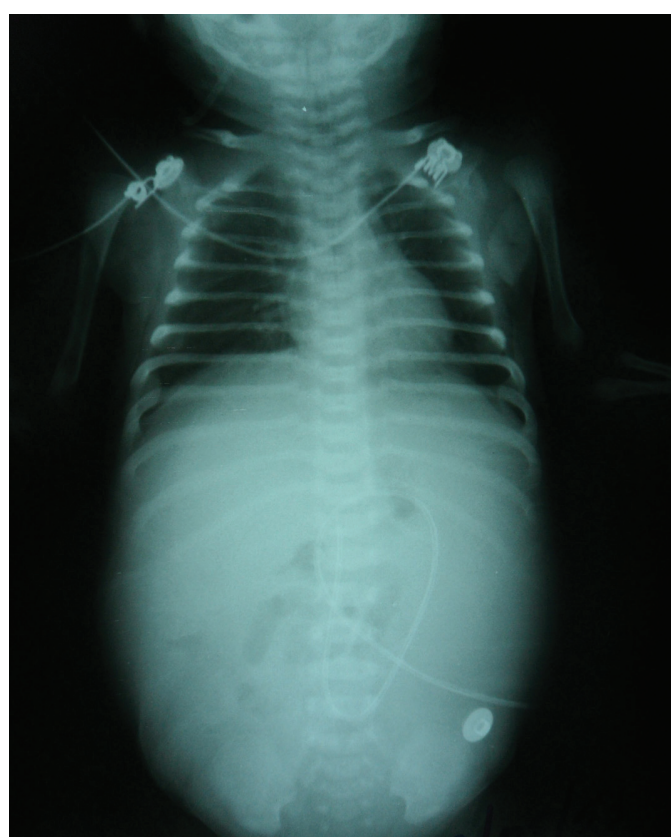

Figure 1. Plain abdominal film on day 11 of life revealed abdominal distension with diffuse increase density and poor definition of the soft tissue shadows.

at day 11 of life. Patient's oxygen saturation was 98 - 99\% during treatment. No surfactant therapy was used.

She was not given any oral fluid till day 10 , and hence she was on Total Parenteral Nutrition (TPN) during this period. On day 10 , enteral feeding was initiated by premature formula $(12 \mathrm{kcal} / \mathrm{oz}) 1 \mathrm{ml}$ every 3 hours and increased to $3 \mathrm{ml}$ on the following day. After feeding, neonate developed abdominal distention, however no gastric residual was seen.

Vital signs were stable. On physical examination, there was marked abdominal distension with hypoactive or reduced bowel sound, with positive fluid thrill and shifting dullness. Confirmatory plain abdominal roenterography showed abdominal distension with diffuse increase density and poor definition of soft tissue shadows; ascites was suspected (Fig. 1). Ultrasonography of abdomen depicted massive ascites with other normal abdominal findings. Baby was on Nil per os/Nil per oral (NPO) with abdominal tapping as a therapy. Twenty milliliters of milky turbid fluid came out while tapping and it was examined. Examination revealed total cell count as 885 cells $/ \mathrm{mm}^{3}$, WBC as 801 cells $/ \mathrm{mm}^{3}$, $\mathrm{RBC}$ as 84 cells $/ \mathrm{mm}^{3}$, PMN as $81 \%$, Monocytes as $1 \%$ and Macrophage as $18 \%$. Ascitic fluid examination revealed no organism found by gram strain and culture. Ascites biochemistry measurement revealed that protein was $0.76 \mathrm{~g} / \mathrm{dL}$, Serum ascites albumin gradient (SAAG) 3.87, (serum Alb = $4.63 \mathrm{~g} / \mathrm{dL}$, serum globulin $=2.8 \mathrm{~g} / \mathrm{dL}$ ), fluid sugar $282 \mathrm{mg} /$ dL (blood sugar 104), fluid LDH $145 \mathrm{U} / \mathrm{L}$, fluid cholesterol $15 \mathrm{mg} / \mathrm{dL}$, fluid triglyceride (TG) 3332 (Serum TG 191).
From days 12 to 17, patient was on NPO with TPN and octreotide $1 \mu \mathrm{g} / \mathrm{kg} / \mathrm{hr}$ extended till 20th day of life. Meropenum was given for 14 days with provisional diagnosis of spontaneous bacterial peritonitis (SBP). However, haemoculture and ascites culture revealed negative growth, which ruled out SBP. On day 18 of life, follow-up echocardiogram revealed closed PDA with no signs of pulmonary hypertension.

On Day 20, feeding test was done with distilled water. No gastric content was observed and there was no progress of ascites, which was established by abdominal circumference comparison. On Day 21 (BW 3550 g), special oral formula was started with Medium Chain Triglycerides (MCT) oil (for lipid), dextrin (for cholesterole) and casein (for protein). She was well tolerated with feed, as there was no abdominal distention. Gradually baby was placed on total oral feeding within 10 days and partial parenteral nutrition (PPN) was weaned off on day 30 of life. On day 40 of life, an extensively hydrolysated casein formula contained MCT (Pregestamil $($ ) was introduced. On follow-up abdominal ultrasound, significant decrease in ascitic fluid was observed with only ascitic fluid accumulation at right hepatic region. As baby was well tolerated on oral feeding, she was discharged from hospital on day 47 with pregestimil and special oral formula (around 50:50 percents of calories) with body weight $3940 \mathrm{~g}$.

At 4 months of age, baby had mild parenchymal liver disease with minimal ascites at right hepatic region by ultrasonography. She was growing well with her body weight and length were about 50 percentiles of Thai children without any previous abnormal findings. Her development was appropriate with the age. She was discharged from nutrition clinic at 9 months of age. At last follow-up she was 11-month of age and had no health problems.

\section{Discussion}

Neonatal chylous ascites is an uncommon condition, hence available data on pathophysiology and therapy of chylous ascites in worldwide literature is scarce. Major reported causes in older children or adult are leaky-lymphatics in abdomen [3], lymphatic atresia or other anomalous of lymphatic drainage, gut malrotation $[3,4]$, obstructions by malignant tumor [5], traumatic causes like surgical trauma [6-8], infective peritonitis [7], cirrhotic liver [8] and genetic mutation [2]. Congenital lymphatic anomaly is more predominant in children [9], while other causes are reported largely in adult. In presented case, neonate was admitted with the symptoms of abdominal distention, however no abnormality was found on physical examination.

Abdominocentesis or paracentesis is used as diagnotherapeutics in the management of chylous ascites [10]. Paracentesis reduces fluid from abdominal cavity, as a treatment modality. In addition, the same fluid is usually examined for 
diagnostic purpose. In demonstrated case report, abdominal tapping led to fluid collection and devised to diagnose. The fluid known as chyle, is usually color free or milky white. The appearance depends on its composition, cellular substance and diet [2]. In this case report; baby was treated with abdominal tapping, led to milky, turbid fluid. Collected fluid in presented case report was deferred than classical ascitic fluid. Collected fluid was having more number of PMNs rather than lymphocytes. Fluid protein was also higher than classical ascitic fluid, showing cirrhotic symptoms. However, increased amount of triglyceride and LDH with exclusion of microorganism through culture and sensitivity inclined towards diagnosis of chylous fluid.

Lymphangiography and lymphoscintigraphy are confirmatory diagnostic means to evaluate the patency of the lymphatic vessels [11]. In our patient, any of this method was not used. Fluid examination and ultrasonography abdomen were only used as diagnostic manner. Thailand being an emerging nation, lymphoscintigraphy and lymphangiography are not commonly used methods.

Only measures used mostly is through dietary management. Restriction in oral fluid and initiation of TPN has been a choice of therapy [12]. MCT oil based diet has been utilized as widely used nutrients due to decrease chyle production efficiency in peritoneal fluid $[1,9,13,14]$. MCT oil is having mechanism to reduce re-esterification through duodenal cell and enters the portal system, so that MCT oil excludes the route of abdominal lymphatics. Usage of Medium chain triglyceride (MCT) diet reduces long chain fat usage, which absorbs and transmits through lymphatic vessels. Reduction of long chain fat usage leads to decrease in lymphatic pressure and lymphatic flow thus helps in diminution of leakage from anomalous lymph [15]. The same happened in presented case, when baby was fed with MCT oil diet, she started improving. In a previously reported study [5], children with chylous ascites were treated with MCT and enhanced protein content diet. Sixty-three percents of the total children were improved significantly with the above mentioned enteral feeding only.

Nonetheless, very small number of reported paediatric cases of chylous ascites was reported with only TPN as primary therapeutic regimen $[12,16]$. In pediatric patients, as lymphatics have not been matured completely, TPN can be an option for avoidance of gut absorption and hence lymph pressure will be reduced. This is a mechanism by which TPN assists in revive of leaky lymphatics [6, 17]. Disadvantages of TPN are intestinal disuse atrophy and usage of central venous line, associated complications. However, none of them happened in our patient luckily.

Reported adult cases also mentioned that surgical correction were only options in cases where conservative approach failed. These surgical approaches include resection of anomalous lymph or ligation of leaky lymphs $[10,18]$. One more approach is perito-neovenous shunt; this is tem- porary means of stoppage of lymph where other ways have defeated. There are also an alternative approaches like fibrin glue and use of somatostatin analogue. Fibrin glue is used as physiological tissue sealants while operation to reduce leakage of vessels. Fibrin glue gives results like final stage of coagulation. It is generally sprayed on leaky parts of vessel as an ancillary treatment [19]. In one of the previously reported case of congenital chyloperitoneum, fibrin glue usage while laparotomy resulted in successful treatment [19]. Octreotide-A somatostatin analogue is a known substance to reduce intestinal absorption of fats. It inhibits intestinal blood circulation by acting on somatostatin receptor, which ultimately reduces lymphatic flows in channels and reduces triglycerides in lymphatic ducts [20]. When octreotide has been combined with TPN, acceptable results have been achieved [8].

In presented case, patient was treated with TPN and octreotide and then fed with MCT oil based diet after 20 days of life. This led to decrease in abdominal girth and reduce in abdominal distention. NPO with TPN might reduced the lymph flow, resulting in reduce lymph leakage and MCT oil based diet could have reduced chyle production. The patient was also given Pregestimil ${ }^{\circledR}$ from day 40 of life, and discharged on pregestimil only. After 9 months of follow-up, baby was normal in growth and she was on normal baby diet without any symptoms of ascites. Triglyceride level was also reduced from 191 on day 12 of life to 69 on 84th day of life.

In conclusion, a rare case of neonatal chylous ascites, developed in the very young child successfully treated with MCT diet, TPN and octreotide. Conservative approach of MCT-based diet and TPN is an effective way of reducing chyle production and lymph flow. Octreotide with MCT oil based diet aids in repair of anomalous lymphatic vessel. There is no standard set of therapeutic approach in newborn. From our experience, neonatal chylous ascites can be successfully treated with diet modification including MCT diet, TPN and octreotide as pharmacotherapy.

\section{Acknowledgement}

We thank Jidapa Stapornchaisit, Thananya Ruengurairoek and Thanit Kamoladisai for their assistance in gathering of patient data.

\section{Grant Support}

The authors have no grant support.

\section{Conflict of Interest}

The authors have no conflict of interest to report. 


\section{References}

1. Cochran WJ, Klish WJ, Brown MR, Lyons JM, Curtis T. Chylous ascites in infants and children: a case report and literature review. J Pediatr Gastroenterol Nutr. 1985;4(4):668-673.

2. Cardenas A, Chopra S. Chylous ascites. Am J Gastroenterol. 2002;97(8):1896-1900.

3. Kelley ML, Jr., Butt HR. Chylous ascites: an analysis of its etiology. Gastroenterology. 1960;39:161-170.

4. Sanchez RE, Mahour GH, Brennan LP, Woolley MM. Chylous ascites in children. Surgery. 1971;69(2):183188.

5. Craven CE, Goldman AS, Larson DL, Patterson M, Hendrick CK. Congenital chylous ascites: lymphangiographic demonstration of obstruction of the cisterna chyli and chylous reflux into the peritoneal space and small intestine. J Pediatr. 1967;70(3):340-345.

6. Leibovitch I, Mor Y, Golomb J, Ramon J. The diagnosis and management of postoperative chylous ascites. J Urol. 2002;167(2 Pt 1):449-457.

7. Lloyd DA. Gastroschisis, malrotation, and chylous ascites. J Pediatr Surg. 1991;26(1):106-107.

8. Shapiro AM, Bain VG, Sigalet DL, Kneteman NM. Rapid resolution of chylous ascites after liver transplantation using somatostatin analog and total parenteral nutrition. Transplantation. 1996;61(9):1410-1411.

9. Browse NL, Wilson NM, Russo F, al-Hassan H, Allen DR. Aetiology and treatment of chylous ascites. $\mathrm{Br} \mathrm{J}$ Surg. 1992;79(11):1145-1150.

10. Lo TS, Chen FP, Chu KK, Soong YK. Successful management of chylous ascites after laparoscopic presacral neurectomy. J Am Assoc Gynecol Laparosc. 1998;5(4):431-433.

11. Matsufuji H, Nishio T, Hosoya R. Successful treatment for intractable chylous ascites in a child using a peritoneovenous shunt. Pediatr Surg Int. 2006;22(5):471-473.

12. Alliet P, Young C, Lebenthal E. Chylous ascites: total parenteral nutrition as primary therapeutic modality. Eur J Pediatr. 1992;151(3):213-214.

13. Chye JK, Lim CT, Van der Heuvel M. Neonatal chylous ascites--report of three cases and review of the literature. Pediatr Surg Int. 1997;12(4):296-298.

14. Liao HB, Hwang RC, Chu DM, Chu ML, Chu CC, Hwang EJ. Neonatal chylous ascites: report of two cases. Zhonghua Min Guo Xiao Er Ke Yi Xue Hui Za Zhi. 1990;31(1):47-52.

15. Biewer ES, Zurn C, Arnold R, Glockler M, SchulteMonting J, Schlensak C, Dittrich S. Chylothorax after surgery on congenital heart disease in newborns and infants -risk factors and efficacy of MCT-diet. J Cardiothorac Surg. 2010;5:127.

16. Smeltzer DM, Stickler GB, Fleming RE. Primary lymphatic dysplasia in children: chylothorax, chylous ascites, and generalized lymphatic dysplasia. Eur J Pediatr. 1986;145(4):286-292.

17. Lee YY, Soong WJ, Lee YS, Hwang B. Total parenteral nutrition as a primary therapeutic modality for congenital chylous ascites: report of one case. Acta Paediatr Taiwan. 2002;43(4):214-216.

18. Laterre PF, Dugernier T, Reynaert MS. Chylous ascites: diagnosis, causes and treatment. Acta Gastroenterol Belg. 2000;63(3):260-263.

19. Antao B, Croaker D, Squire R. Successful management of congenital chyloperitoneum with fibrin glue. J Pediatr Surg. 2003;38(11):E7-8.

20. Collard JM, Laterre PF, Boemer F, Reynaert M, Ponlot R. Conservative treatment of postsurgical lymphatic leaks with somatostatin-14. Chest. 2000;117(3):902905 . 